\title{
Gateway Diversity for a Future High Throughput Satellite System
}

\author{
Argyrios Kyrgiazos* Barry Evans ${ }^{\dagger}$ Paul Thompson $^{\ddagger}$ \\ Centre for Communication Systems Research, University of Surrey, Guildford, GU2 7XY United Kingdom. \\ Mathieu Dervin ${ }^{\S}$ Cedric Baudoin ^ Benoit Garnier $\|$ \\ Thales Alenia Space, Toulouse, 31100 France.
}

\section{Nomenclature}

$\begin{array}{ll}C /(N+I) & \text { Carrier to Interference plus Noise ratio } \\ A C M & \text { Adaptive Coding and Modulation } \\ I X P & \text { Internet Exchange } \\ \text { OSI } & \text { Open Systems Interconnection model } \\ \text { POP } & \text { Point of Presence }\end{array}$

\section{Introduction}

The continuous increase of traffic demands for satellite networks motivates the evolution of the telecommunication satellite technology towards wider channels and multiple beam operation with frequency re-use across the coverage. This is made possible by the use of higher frequency bands. Recent research projects ${ }^{1,2}$ have investigated multi-beam coverages with more than 200 user beams operated in Ka band, to offer very large data throughputs over Europe. Since 2012, the European Commission project Broadband Access via integrated Terrestrial and Satellite systems (BATS) has explored a similar concept based on a dual satellite solution offering around 302 user beams over EU27 and Turkey, targeting 2020 timeframe, see Figure 1. In all these systems, so as to maximize the user link capacity, the whole civil band allocated to Fixed Satellite Services (FSS) in Ka band $(20 / 30 \mathrm{GHz})$ is dedicated to the user links. The feeder links thus have to be operated in another band. An attractive option is to rely on Q/V bands $(30 / 40 \mathrm{GHz})$ to provide the gateway-to-satellite links. Despite the large available bandwidths in $\mathrm{Q} / \mathrm{V}$ band (5 GHz in each direction), the very large user aggregated bandwidths are required to implement several tens of gateways to provide the necessary capacity, Figure 2(a).

Considering that the feeder links operate in $\mathrm{Q} / \mathrm{V}$ bands, they are subject to severe precipitation fades. As each gateway will carry very high capacity, it is essential to ensure very high availabilities for the feeder links, typically greater than $99.9 \%$. In order to do so, spatial diversity is required, since Adaptive Coding and Modulation (ACM) is unable to cope with the fade dynamics encountered at these high frequency bands. In an effort to reduce the total number of gateways in the system, the concept of N+P Smart Gateways has emerged which adds P redundant per $\mathrm{N}$ active gateways ${ }^{3}$. In this paper, we discuss the methodology to design and optimise the diversity scheme for a scenario involving 302 user beams and 50 gateway earth stations ( 25 gateway sites) ${ }^{4}$, part of the overall system design methodology is shown in Figure 2(b). The paper structure is the following: first we introduce the main issues related to the diversity, such as selection of gateways sites and its fading statistics and dynamics. The sites were selected in an effort to maximise the inter-sites distance and for favourable propagation conditions. For the evaluation of the fading statistics, the ITU-R P618-10 recommendation was used. We then proceed with the definition of propagation margin. The clustering of gateways not only depends on the clustering scenario but also on the complexity added in the ground segment and on the complexity of the on-board switching matrix. The envisaged solutions include the number of redundant gateways, switching threshold and the sizing of the feeder link. Thus, the final selection needs to take into account all the above parameters. Herein, we evaluate the availabilities that can be achieved for different solutions and the impact of each solution on the overall system design. Finally, a hysteresis period is also introduced in the

\footnotetext{
${ }^{*}$ Researcher, CCSR, University of Surrey, non-member.

${ }^{\dagger}$ Professor, CCSR, University of Surrey, AIAA Senior Member.

$\ddagger$ Senior Researcher, CCSR, University of Surrey, AIAA Senior Member.

$\S$ Satcom System Engineer, Thales Alenia Space, non-member.

`Satcom System Engineer, Thales Alenia Space, non-member.

"Satcom System Engineer, Thales Alenia Space, non-member.
} 


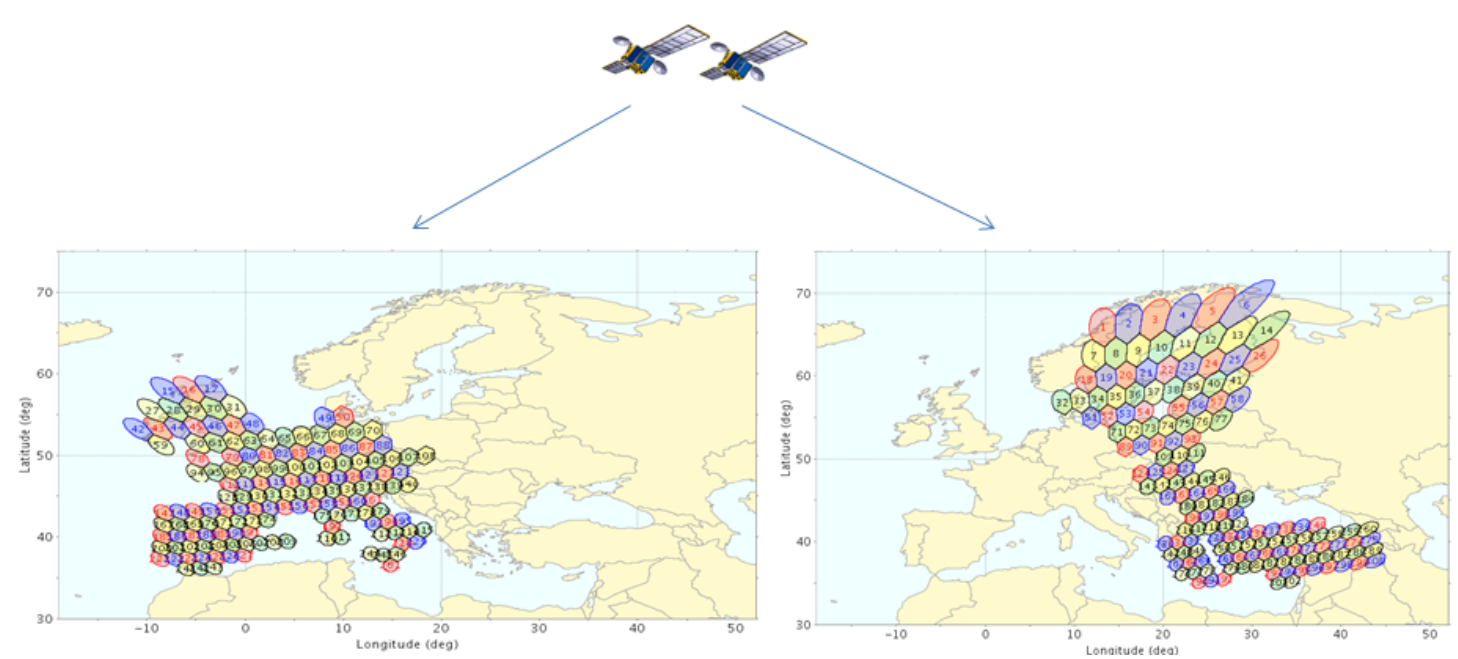

Figure 1. BATS beam pattern, 302 spot beams, 2 collocated satellites

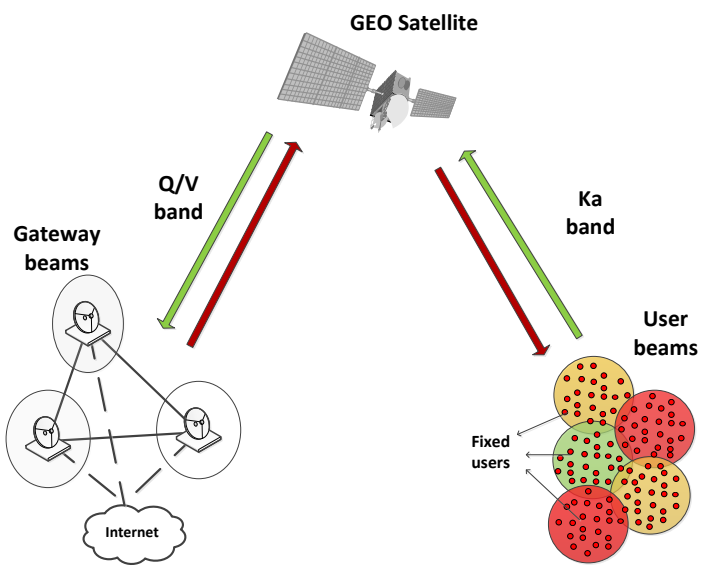

(a) Architecture of a BATS satellite system

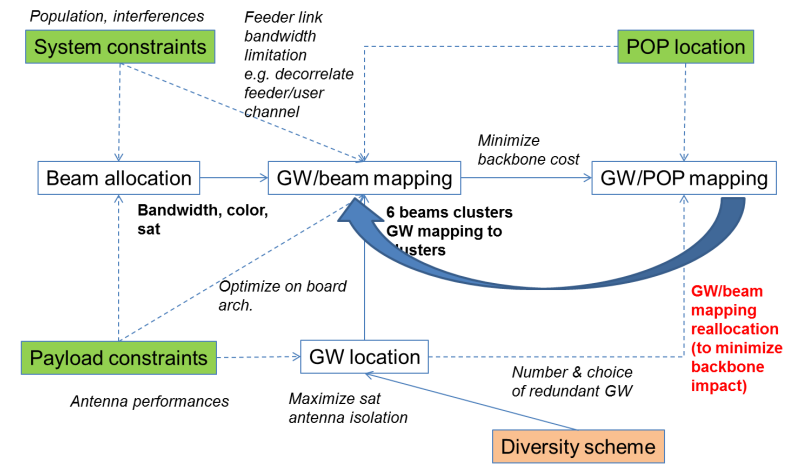

(b) Methodology for satellite system and backbone design

Figure 2. BATS satellite system design

switching decision in order to alleviate very frequent handovers due to the large numbers of fades of short duration.

\section{State of the art - Diversity schemes}

$\mathrm{In}^{5}$, the Smart gateway scheme was presented and discussed for the first time. It can be said that Single Site Diversity is a special case of Smart Gateway diversity. The author illustrates the benefit of the interconnection between such gateways. He makes a first level evaluation of the performance and concludes that the system's availability can achieve very high percentages for lower individual gateway availabilities. These results do not take into consideration any correlation issues between the gateways because they are deemed totally uncorrelated. $\operatorname{~}^{6}$, a preliminary assessment of this macro-diversity scheme was presented. It is shown that this mechanism enables the system to preserve high availability without impacting significantly the satellite payload. Two aspects of this scheme were examined, called $\mathrm{N}+0$ and $\mathrm{N}+\mathrm{P}$ with $\mathrm{P}$ representing the redundant site-gateways. These two mechanisms are compatible with bent pipe satellite payloads. Formulas for the evaluation of the outage probability are proposed, considering no correlation amongst the gateway sites. $\mathrm{In}^{3}$, a mathematical formulation for the outage probability of the smart gateways was presented.

The Smart Gateway diversity scheme employs a number of Gateway Earth Stations (GES) which are interconnected via terrestrial links to form an agile routing of feeder link data that can be used in a diversity manner to combat fades on the gateway to satellite links ${ }^{5}$. The approach is depicted in the Figure 2(a).

The use of networked or "Smart Gateway" diversity can eliminate the problems for more costly single site diversity. Smart diversity techniques exploit the spatial diversity existing between the gateways in order to relax the requirements on the system margins, without requiring additional earth stations. In the Smart diversity scheme: 
- A UT (User Terminal) beam is connected not just to one gateway but to a pool of gateways.

- If a gateway experiences very deep fades, then its traffic can be routed to another gateway that has available capacity

Some additional cost will be required for the interconnection between the gateways (fibre optic links at Gbps). As the distance between gateways will be greater than in typical single site diversity systems, rain correlation must be determined over a wider area. Since all of the gateways belong to the same network, some extra provision has to be taken in order to guarantee that the time that both sites experience high fades is minimized. In addition, the interference factor is always present, but in this case extra provision has to be taken for this issue. For a given beam, the higher the number of gateways in the pool the lower the fading margin required and the lower the fraction of capacity lost when a gateway link becomes unavailable. User-link beam radio resources need to be assigned to several gateways. The management of traffic flow can be performed by a centralized Network Control Centre (NCC) to which all gateways are connected. A decentralized cooperative approach between the gateways is rather too complex to be employed and probably limited by the network size. The whole gateway network consists of $N$ active gateways and $P$ redundant. In this scheme, a UT beam is served by just one gateway at a time and switches to a redundant gateway when faded ${ }^{6}$.

\section{$\mathrm{N}+\mathbf{P}$ diversity}

In a system where only one redundant station exists, if two of the gateways become unavailable, then only the UT beams from one gateway will be served by the redundant gateway. This scheme takes advantage of the statistical decorrelation of the gateway rain events. As was mentioned earlier, by spreading the gateways over large distances, it is unlikely that more than two gateways will experience deep fades.

The number of redundant gateways needed to ensure a certain feeder link availability is subject to availability levels required and to the network size ${ }^{3}$. In terms of payload complexity, the logic is the same as for the single site diversity, with the only difference being that the redundant gateway beam can be switched to more than one transponder. The latter implies that the connections on the payload are also increased.

\section{System model for global feeder link availability}

For a user link to be in outage due to feeder link fade, the gateways or the pool of gateways that serve it have to be in outage. For the $\mathrm{N}$-active scheme, all the gateways need to be in outage simultaneously. For the $\mathrm{N}+\mathrm{P}$ scheme, a methodology to evaluate the feeder link availability is proposed in ${ }^{3}$. However, this methodology assumes equal outage probabilities for each gateway, which is not the case in practise, where the outages differ. Herein, the methodology from $^{3}$ is extended to consider unequal outages probabilities.

The probability of having $k$ gateways in outage, out of $N$ gateways, can be derived for all the possible combinations $^{3}$. It is known that there are $\left(\begin{array}{c}N \\ k\end{array}\right)=\Lambda$ possible combinations in a system where $N$ gateways exist. These combinations are the solutions of the following equation:

$$
x_{1}+x_{2}+\ldots+x_{N}=k
$$

where $x_{i}$ is a binary variable that indicates if the $i^{t h}$ is in outage. If $x_{i}=1$ then the $i^{\text {th }}$ gateway is in outage, while if $x_{i}=0$ then it is not. Lets consider as $X^{l}$ the $l^{\text {th }}$ solution of equation (1), where $l=1 \ldots \Lambda$. Thus we have:

$$
\begin{array}{r}
P(\mathrm{k} \text { gateways to outage })=P(\text { set of k gateways in outage }) \times P(\text { set of N-k available gateways })= \\
\qquad \sum_{l=1}^{\Lambda}\left(\prod_{j=1}^{N} p_{j}^{X_{j}^{l}}\right) \cdot\left(\prod_{j=1}^{N}\left(1-p_{j}\right)^{\left|X_{j}^{l}-1\right|}\right)
\end{array}
$$

The above equation is known as Poisson-Binomial distribution. Its pdf describes the numbers of successes in $\mathrm{N}$ independent trials when the individual probabilities of success are different across trials. To recall that in the binomial distribution, the individual probabilities of success are constant across trials ${ }^{7}$.

In our scenario, the system consists of $\mathrm{N}+\mathrm{P}$ gateways. The outage probability of the $i^{\text {th }}$ gateway is $p_{i}$. The number $\mathrm{k}$ of number of gateway in outage can be written as the sum $Y=X_{1}+X_{2}+\ldots+X_{N}$ of N mutually independent random variables $X_{k}$ with $\operatorname{Pr}\left\{X_{k}=0\right\}=1-p_{k}$ and $\operatorname{Pr}\left\{X_{k}=1\right\}=p_{k}$. The distribution of the sum Y of these random variables, the Poisson-binomial pdf is then given by the linear convolution of the distributions of the $X_{k} s$;

$$
\begin{aligned}
\operatorname{Pr}\{Y=0\}= & \left(1-p_{1}\right)\left(1-p_{2}\right) \ldots\left(1-p_{N+P}\right) \\
\operatorname{Pr}\{Y=1\}= & p_{1}\left(1-p_{2}\right) \ldots\left(1-p_{N+P}\right)+ \\
& \left(1-p_{1}\right) p_{2}\left(1-p_{3}\right) \ldots\left(1-p_{N+P}\right)+ \\
& +\ldots+\left(1-p_{1}\right)\left(1-p_{2}\right) \ldots\left(1-p_{N+P-1}\right) p_{N+P} \\
& 3 \text { of } 10
\end{aligned}
$$




$$
\begin{aligned}
& {[\operatorname{Pr}\{Y=0\} \operatorname{Pr}\{Y=1\} \ldots \operatorname{Pr}\{Y=N+P\}]=} \\
& \quad=\left[p_{1} 1-p_{1}\right] *\left[p_{2} 1-p_{2}\right] * \ldots *\left[p_{N+P} 1-p_{N+P}\right]
\end{aligned}
$$

For convenience, let us define as $\operatorname{Pr}\{Y=k\}=P_{k}$ If we consider the X-transform (similar to Z-transform $X=z^{-1}$, we use the symbol $\mathrm{Z}$ ) of the above expression, we have that:

$$
\begin{aligned}
& P_{0}+P_{1} z+P_{2} z^{2}+\ldots+P_{N+P} z^{N+P}= \\
& =\left(p_{1} z+1-p_{1}\right)\left(p_{2} z+1-p_{2}\right) \ldots\left(p_{N+P} z+1-p_{N+P}\right) \\
& =\alpha\left(z-s_{1}\right)\left(z-s_{2}\right) \ldots\left(z-s_{N+P}\right)
\end{aligned}
$$

where

$$
\alpha=\prod_{i=1}^{N+P} p_{i}
$$

and

$$
s_{i}=-\left(1-p_{i}\right) / p_{i}
$$

Following the methodology described in ${ }^{7}$, a closed form expression can be derived for $P_{k}$.

$$
\begin{array}{r}
P_{k}=\operatorname{Pr}\{Y=k\}=\operatorname{Pr}\{\mathrm{k} \text { gateways in outage }\}= \\
=\sum_{n=0}^{n=N+P}\left\{e^{-j 2 \pi n k /(N+P+1)} \prod_{m=1}^{N+P}\left\{p_{m} e^{j 2 \pi k /(N+P+1)}+\left(1-p_{m}\right)\right\}\right\} /(N+P+1)
\end{array}
$$

Let us consider the configuration in which every user beam is served by one gateway and for every $N$ gateways $P$ redundant gateways are added as provision. For a user beam to loose service (due to a feeder link issue), the regular gateway needs to become unavailable and the $P$ extra to be unavailable as well (either dedicated to others beams or experience deep fades). According to ${ }^{3}$, the probability of a user inside a spot beam to be in outage is the probability of the gateway that serves it to experience the outage (or deep fades), plus the probability to belong to one of the $\mathrm{k}$ gateways that experience outage.

$$
\begin{array}{r}
P_{\text {outage }}=\frac{1}{N} \operatorname{Pr}\{\mathrm{P}+1 \text { gateway to outage }\}+ \\
+\frac{i}{N} \operatorname{Pr}\{\mathrm{P}+\mathrm{i} \text { gateways to outage }\}= \\
\sum_{i=0}^{N} \frac{i}{N} \operatorname{Pr}\{\mathrm{P}+\mathrm{i} \text { gateways to outage }\}
\end{array}
$$

\section{Gateway diversity - Requirements \& Design}

The requirements of the diversity scheme are related to the availability of the feeder link to be above a specific value, for example $99.9 \%$, and the reduction it causes to a beam's capacity to be less than a desired value, $25 \%$. For a gateway to be able to provide a specific percentage of its nominal capacity for $\mathrm{p} \%$ of time, it means that the margin included in the link budget and any uplink power control (UPC) being applied is able to compensate for the total atmospheric attenuation for $\mathrm{p} \%$ of time. In Figure 3(a), the relation of propagation margin with UPC and system margin is illustrated. Note that the use of rain only or total atmospheric attenuation depends on how the link budgets are designed. The nominal gateway remains active for the percentage of time which corresponds to $\mathrm{p} \%$.

The system margin is defined as the maximum allowable degradation of the feeder link $\left(\Delta_{F W D}\right)$, assuming ideal (clear weather) UT link, given that the feeder link will still be able to provide a specific percentage of its nominal capacity to the users. Neglecting intermodulation noise and assuming that the satellite HPA is operated at constant operating point, it is well known that $C /(N+I)_{\text {Total }}=\left(C /(N+I)_{\text {up }}^{-1}+C /(N+I)_{\text {down }}^{-1}\right)^{-1}$. From this relationship, $\Delta_{F W D}$ can be calculated. Knowing $\Delta_{F W D}$ and UPC level, the maximum allowable rain (or total atmospheric) attenuation can be calculated for every site such as not to degrade a beam's capacity more than a desired percentage, Fig. 3(b).

Thus if it is required to design a gateway such as to be available for $\mathrm{p} \%$ of time, then the total atmospheric attenuation exceeding $\mathrm{p} \%$ for gateways site can be calculated according to:

$$
\text { Propagation margin } \mathrm{p} \%=\Delta_{F W D}+\mathrm{UPC}
$$

and appropriate $\Delta_{F W D}$ and UPC can be chosen. 


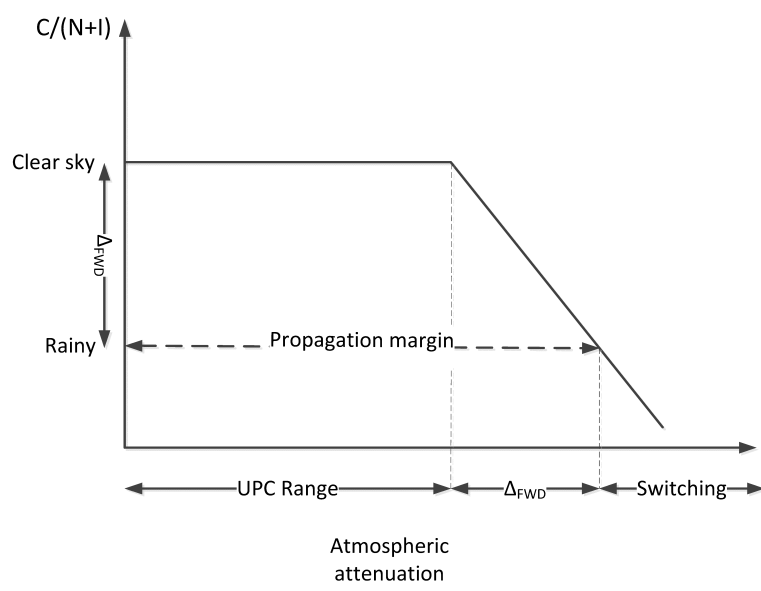

(a) Definition of propagation margin

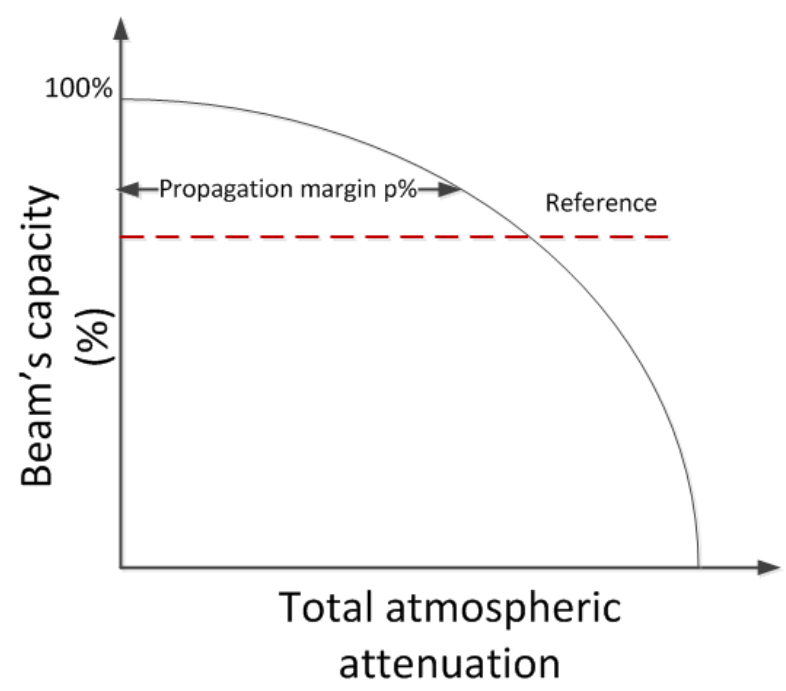

(b) Beam's capacity versus total atmospheric attenuation

Figure 3. Propagation margin for the feeder uplink

\section{A. Design Challenges}

Considering the complexity and cost of a gateway at $\mathrm{Q} / \mathrm{V}$ bands which are further increased compared with a state of the art gateway at Ka band, we need to design the ground segment with the fewest possible gateways. In the case of two collocated satellites, there is the possibility to also collocated two gateway earth stations, with each GES pointing to one satellite. However, the impact of the backbone design needs to be taken into account during the design process which may affect the final decision. The design and the optimisation of the gateway diversity scheme is a complex task. The main parameters are the following

- overall number of gateways in the system

- backbone terrestrial links

Regarding the $\mathrm{N}+\mathrm{P}$ diversity scheme, it can be considered that the gateways are interconnected or separate. This has an impact on:

- the cost of the terrestrial links

- the switching matrix on board the satellite

- handover/switching process

Considering that all the gateways are interconnected with each other, the number of terrestrial links as well as the cost increase significantly, as each link needs to be active 24/7. On the other hand, the handover process can then be simplified by creating a tunnelling between the primary and the secondary gateway, the traffic can be routed easily. If no interconnection is assumed amongst the gateways, then the cost of the terrestrial links is reduced significantly, but the handover process becomes more complex. In this case, the traffic will need to be redirected via the redundant gateway. Considering the volume of traffic that needs to be handed over ( $\succ 10 \mathrm{Gbps}$ ), it is difficult to engineer it such as to be transparent to the users.

Furthermore, Figure 4 illustrates the impact of the diversity on the backbone topology in the case of collocated satellite hubs. In this example, the nominal hub in France serves beams in France and Germany for the West satellite and in Romania for the East satellite. In the case where the fading exceeds the margin of the nominal feeder, the redundant satellite hub is selected to serve those beams.

\section{B. Cost of backbone design}

The cost of the terrestrial links include the cost for the connection gateway to Internet eXchange points (IXP), IXP to IXP and IXP to Point of Presence (POP),8. Usually, the POPs are located close to the IXP, so this cost can be considered negligible compared to the rest. Figure 5(a) shows the IXP locations and interconnections in Europe. Each cost figure is assumed to be the result of the sum of : the access transport (GW IXP) cost corresponding to the edges of the network managed by a local, national internet service provider. We are using a combination of a flat charge and a distance charge (applied only for non-metropolitan access, i.e. for distance of 25 kilometers or more). 


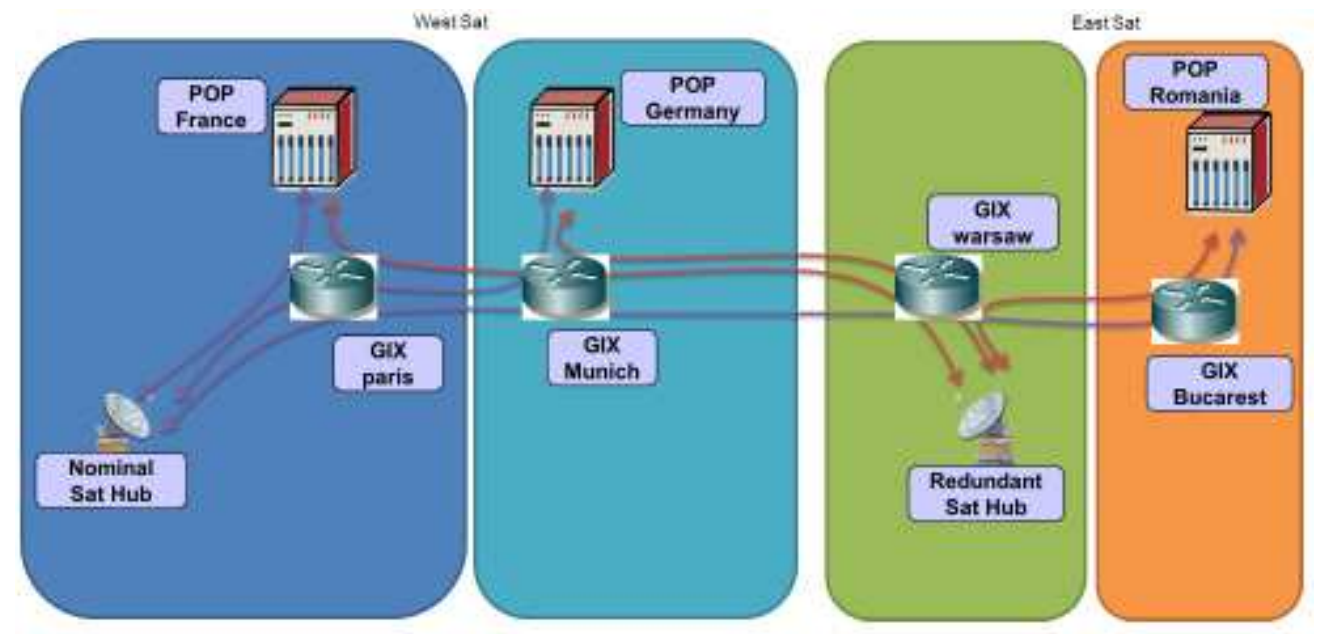

Figure 4. Diversity impact on topology

We are using a linear distance charge computation (cost $=$ Distance Rate $\mathrm{x}$ Distance). In addition, the Distance Rate increases as a function of required bandwidth, also in a close to linear way. the peering transport (IXP - IXP) cost corresponding to the backbone edges of the network, assumed to be managed by a single CDN operator for each satellite subsystem. Based on peering related principles, we are using a combination of a flat charge (corresponding to the minimum volume required to motivate a sensible peering agreement) and a linear distance charge which rate decreases as a function of the required bandwidth, in a close to exponential way.

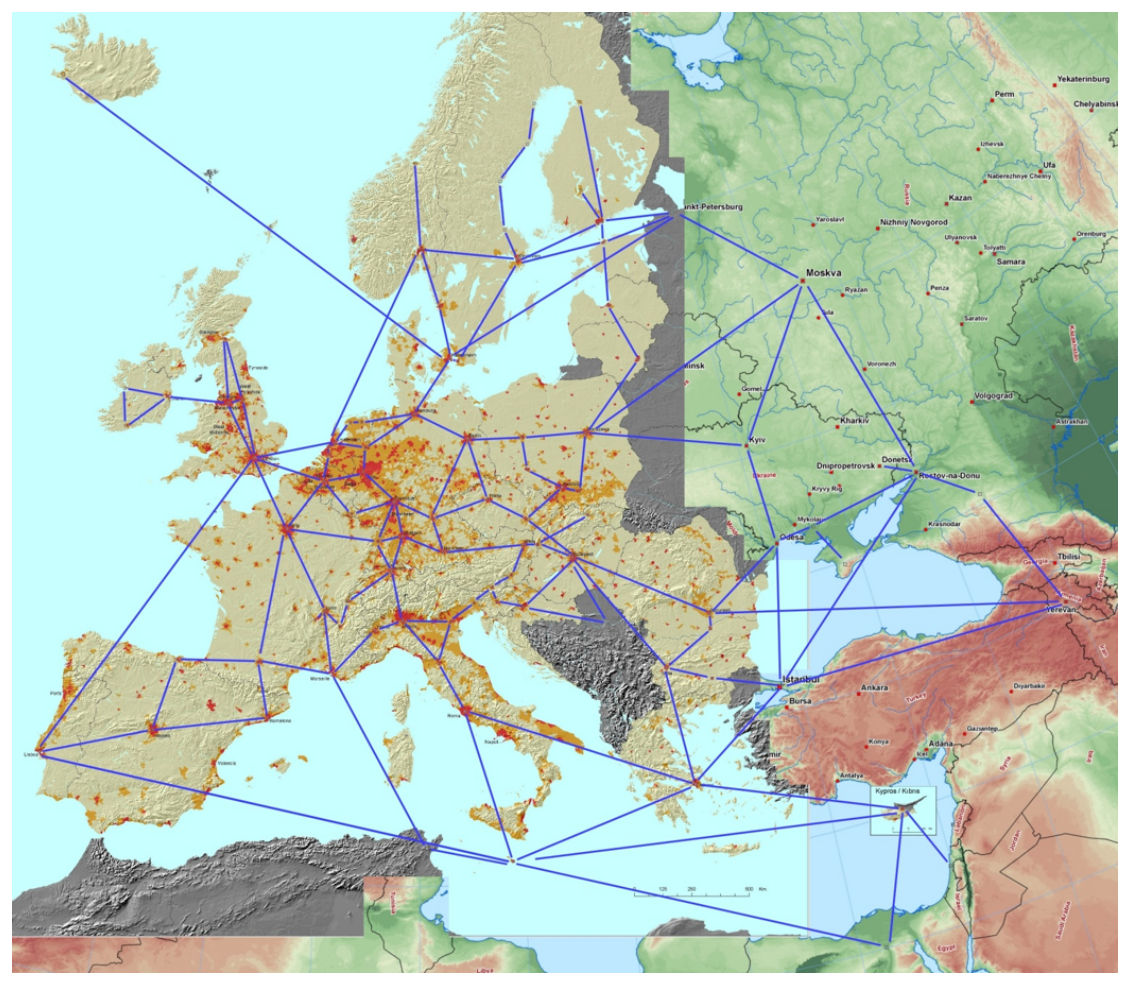

Figure 5. IXP in Europe

However, it is possible to optimise the backbone design only for one cluster of user beams as can be seen in Figure 6(b). For example, a site in Istanbul with two gateways, where each gateway serves a cluster in each of the coverage areas. The distance to a country's POP for the cluster to EAST is shorter than the distance to the POPs for the cluster in WEST. This results in large distances to connect the gateways to the POP of the served countries. $\operatorname{In}^{8}$, the authors describe a methodology how to optimise this design. 


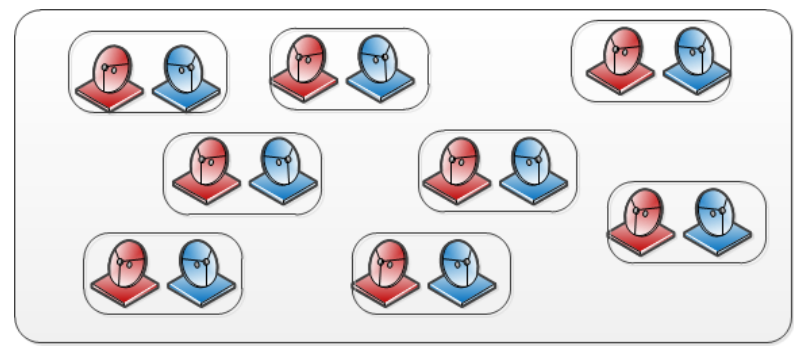

(a) Example of collocated gateways, ${ }^{4}$

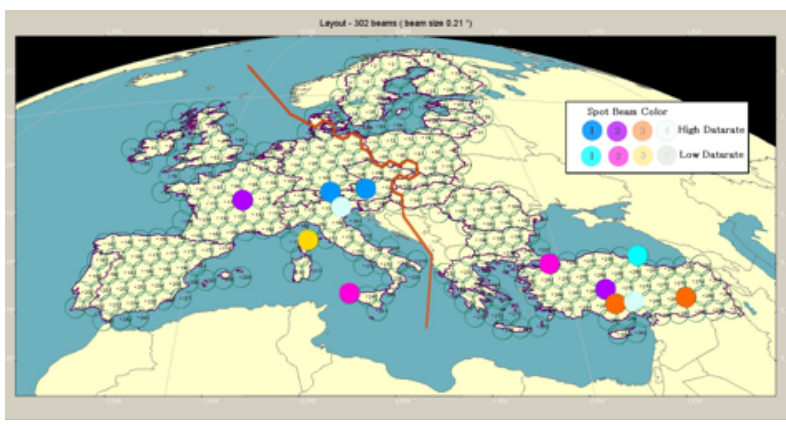

(b) Example of beam gateway mapping - 2 collocated satellites

Figure 6. Example of collocated satellites

\section{Selection of active and redundant gateways}

The choice of the gateway sites is of paramount importance. The sites need to be selected in such a way as to minimise the terrestrial link costs; on using where possible existing teleports and maximising the distance such as to minimise the satellite antenna C/I degradation. Regarding the selection of the active and redundant sites, two possible approaches can be applied.

- to consider as active gateways the $\mathrm{N}$ gateways with the best propagation conditions, and the rest as the $\mathrm{P}$ redundant. In this way, we can reduce the switching frequency, as this depends on the outage of the active gateways.

- to consider the P gateways in such a way as to optimise the backbone design.

The latter approach is expected to save cost and form a more optimal backbone design, while the former will reduce the frequency of the handovers. The merits of the first approach perhaps outweigh those of the second.

\section{Handover's analysis}

As soon a fade is detected or predicted and a switching of traffic from a active gateway to a redundant one is decided, then information about the served users is being transferred to the redundant gateway. As described before no interconnection amongst the gateways is assumed, thus the users need to be handovered to the redundant gateway. In order to alleviate the internal rerouting issue in the diversity backbone or the need to transfer TCP-PEP context transfer from one satellite hub to another, the reference architecture is based on a centralized PEP located. It is described in Figure 7.

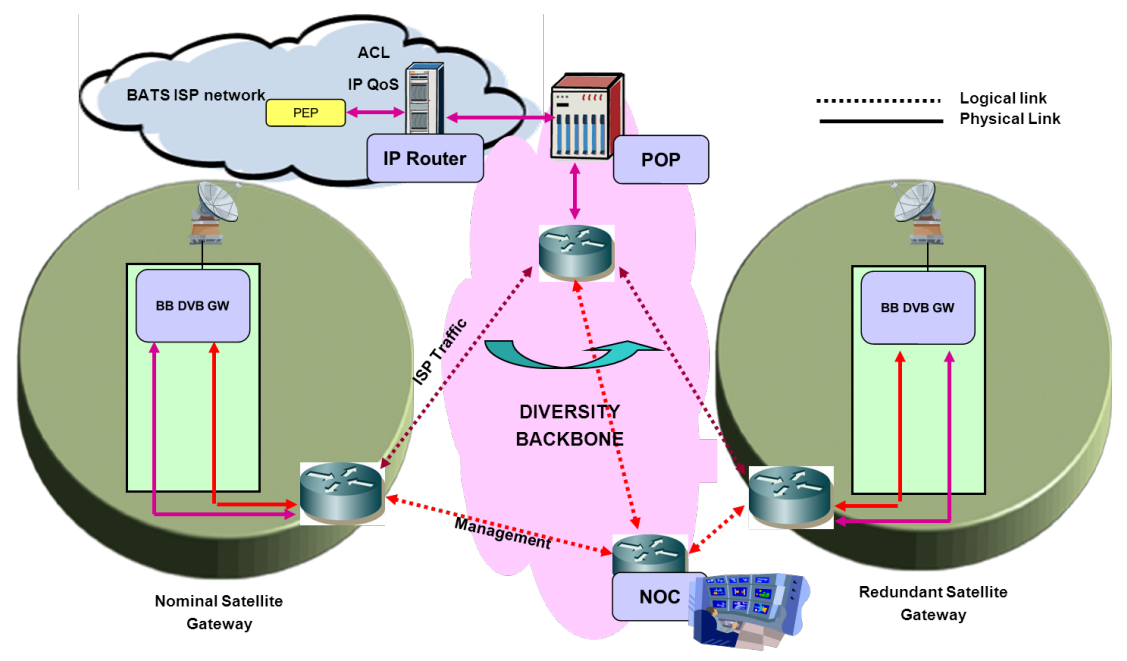

Figure 7. Centralized PEP and IP router

This architecture offers several major advantages: First, from a network architecture perspective, it has clear separation between the IP and the Layer 2 (MEF or MPLS) domains. This leads to an architecture close to the xDSL architecture where the edge IP router (BNG) in charge of the IP QoS and access control with ACL is connected to an Ethernet (or ATM) aggregation network towards the DSLAM equipments. The second benefit is obviously to simplify 
the GES architecture and to possibly reuse existing ISP equipments (e.g. QoS enforcement) and thus to decrease the cost of the ground segment. Finally, TCP connection are always accelerated even during the hand-over phase since there is no need to transfer PEP information from the nominal to the redundant GES. ACL \& IP QoS are also centralized with the same advantage, allowing reducing the preparation time for switching. Finally, the switching itself relies on fast redundancy schemes defined for Metro Ethernet or MPLS networks. For Metro Ethernet Networks, Ethernet Ring Protection (ERP) switching (ITU G8032) is a fast protection scheme based on redundancy links loops setup by management means. It has been designed for ring topologies (e.g. SDH) but a version for chained topologies has been also specified. MPLS Fast Reroute (FRR) can provide protection against link breakage in a LSP by a backup path that circumvents the broken links. The principle for HTS system is to simulate link failure thanks to management means to initiate the Gateway Hand-Over. These solutions allows fast recovery time equivalent to SDH (around 50 $\mathrm{ms})$.

\section{Design Results}

In recent studies ${ }^{1,2,4}$, solutions of collocated smaller GEO satellites have been investigated rather than a single big GEO, as shown in Figure 1. In this case, it is possible to reduce the number of gateway sites, by collocating the gateways, for example two gateway per site with each gateway looking at a different satellite, see Figure 6(a) in which red and blue indicate the two satellites providing EAST or WEST coverage. Then a gateway site will serve a cluster of beams in the WEST side and a cluster of beams in the EAST. Then, less gateways sites are required, and the angular separation distance between the gateways can be increased resulting in better satellite C/I. The drawback of this approach is that each gateway site must be interconnected with Internet Service Provider (ISP) points of presence (PoP) spread over both west and east coverage areas. Including the backbone interconnection cost into the objective function had led us to define an alternate scenario : in the western / eastern coverage areas, the gateway sites involve a single earth station dedicated to the related satellite, while dual sites are maintained in the central zone.

In the case of the BATS network ${ }^{4}$ we have selected the gateway sites based upon minimising the terrestrial link costs; on using where possible existing teleports and maximising the distance such as to minimise the satellite antenna C/I degradation. Figure 8(a) and Figure 8(b) show the selected gateway sites. The considered clustering options are;

1. 25 active gateways $+\mathrm{P}(1 / 2 / 3 / 4)$ redundant

2. 12 active gateways (EAST, WEST) +1 redundant $\& 13$ active gateways (CENTRE) +1 redundant

The redundant gateways are determined based on the propagation conditions at their sites. The gateway sites with the most favourable propagation conditions are chosen as the nominal gateways and the rest as the redundant ones.

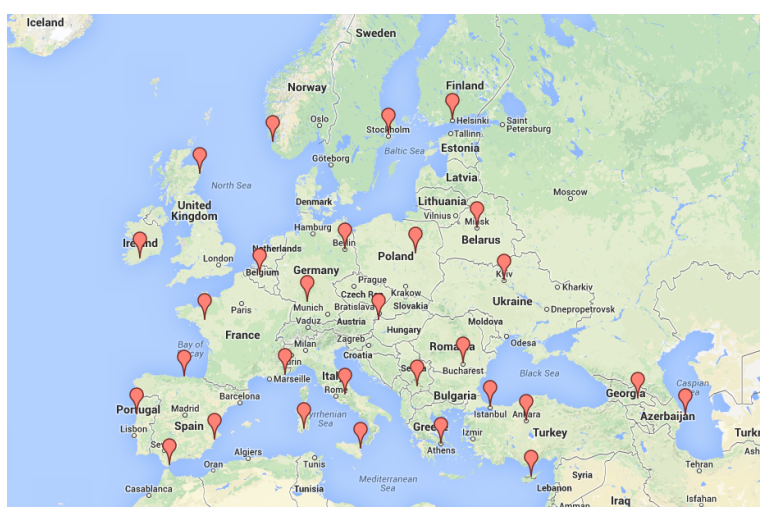

(a) BATS 29 dual gateway sites

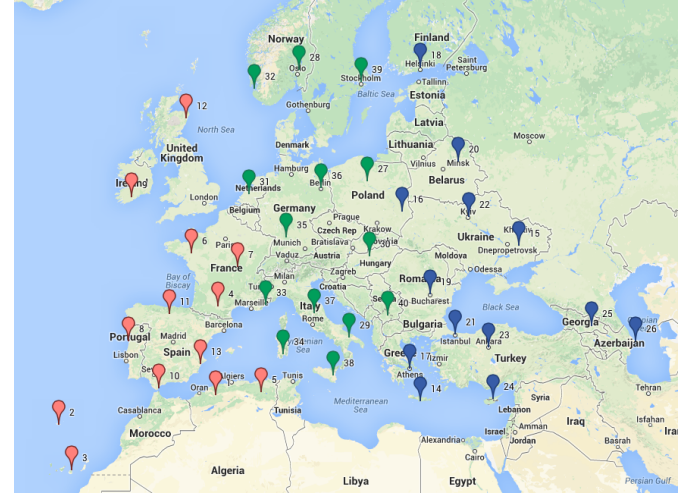

(b) BATS single/dual gateway sites, red markers indicate the WEST, blue the EAST, and green the dual gateways in the CENTRE.

Using the methodology described in section III., we can compute the global feeder link availability for different total atmospheric attenuation thresholds, using the ITU-R P618-10 recommendation. Figure 8 shows the minimum propagation margin that is required to ensure $99.9 \%$ availability for all the clustering scenarios. It can be seen that the $25+1$ and the $37+3$ (alternative) scenarios can provide the desired availability of $99.9 \%$ for a attenuation margin less than $14 \mathrm{~dB}$. Figure 9 illustrates the system capacity reduction (\%) versus the total attenuation margin. It is implicit that the worst reduction of system capacity occurs for the clustering scenario $25+1$, which is less than $25 \%$.

\section{Switching decision algorithm}

An important aspect of the diversity scheme of the ground segment is the switching (routing) rate that the traffic is re-routed from a gateway with deep fades to another one free of fades or with less fade. In the single site diversity 


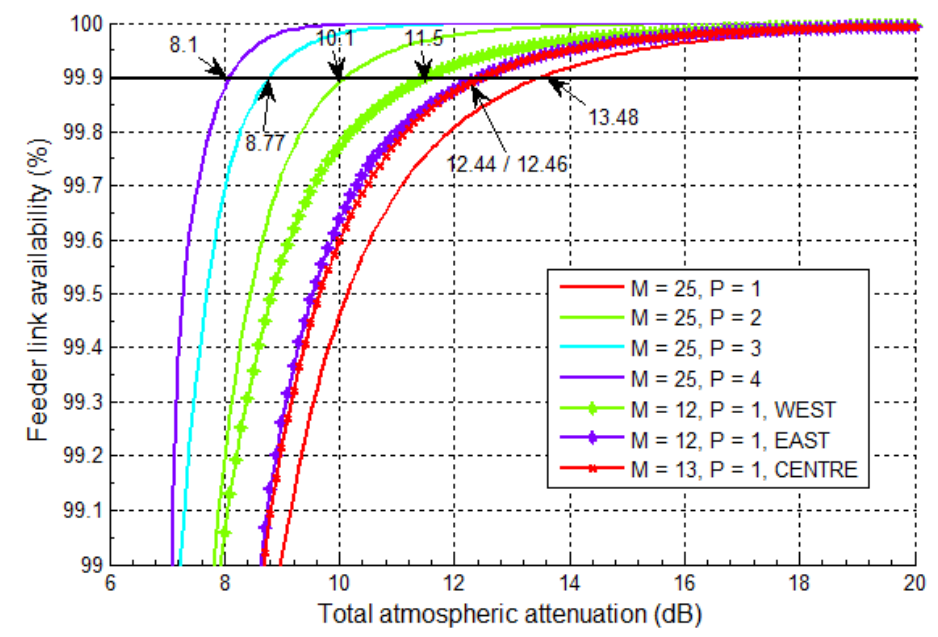

Figure 8. Global feeder link availability versus total atmospheric margin, all scenarios

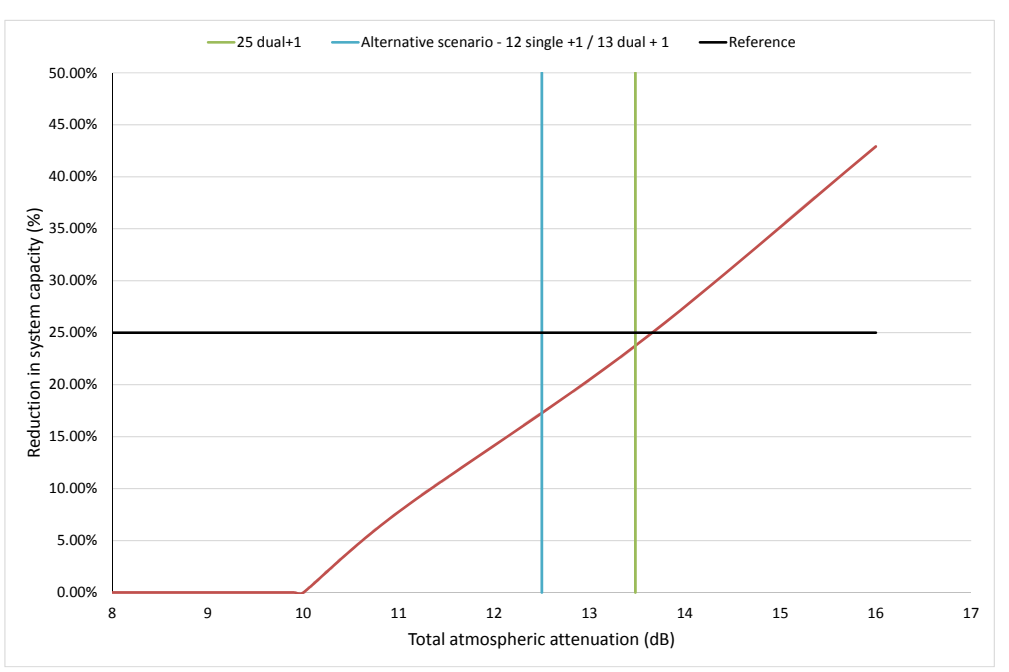

Figure 9. Reduction of system capacity $(\%)$ versus total atmospheric margin

scheme $^{9}$, as soon as a fade is observed and the atmospheric conditions in the redundant site are better, the traffic is switched to the redundant gateway. This logic and the definition of the propagation margin are illustrated in Figure 3(a). First, UPC is applied and then a tolerance $\Delta_{F W D}$ in reduction of the $\mathrm{C} /(\mathrm{N}+\mathrm{I})$ is allowed before switching. In the case of $\mathrm{N}+\mathrm{P}$ scheme, if a gateway experiences deep fades, its traffic is re-routed to a free gateway from the $P$ redundants. If all $P$ redundant gateways are occupied then ACM can be used to compensate for the fades in the feeder link. It is desirable to minimise the switching rate as it has strong impact on the upper layers of OSI model (network, transport etc). It is not wise to re-route the traffic from a gateway to another as soon as a fade is observed, but an hysteresis period is used to avoid frequent re-routings. The purpose of this hysteresis period is to ensure that the instantaneous observed fade is actually due to rain and not any fast variations of any form in the received power. Especially in $\mathrm{Q} / \mathrm{V}$ bands or higher, these events are much more frequent.

A great variation of the $\mathrm{C} /(\mathrm{N}+\mathrm{I})$ value across the sites is expected due to different propagation characteristics. Lets consider that the threshold of the $\mathrm{C} /(\mathrm{N}+\mathrm{I})$ is $10 \mathrm{~dB}$, and if this threshold is exceeded then an "outage" will occur. In Fig. 10, the number of outage events in relation to the outage duration is shown, using ${ }^{10}$.

It is clear that a large number of outages will last just a few seconds and this varies from site to site. Then the 


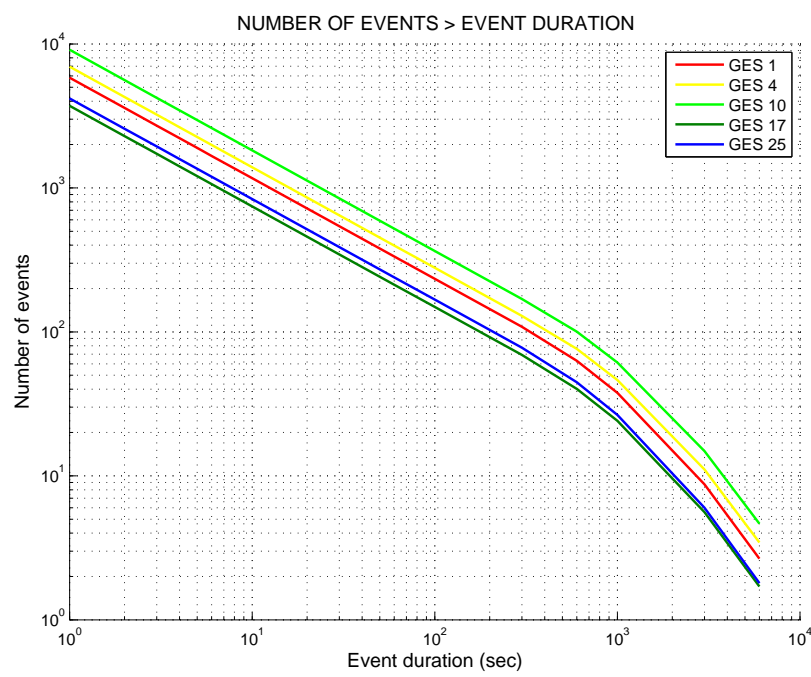

Figure 10. Example of fade duration statistics

question as to whether a switching needs to take place immediately after an outage is detected is posed. Therefore, the addition of an hysteresis period can be considered in order to account for these short duration outages. Furthermore, the use of a weather radar seems appropriate as each gateway earth station can predict more accurately the fade duration using the radar's information.

\section{Conclusion}

In this paper, we discuss the methodology to design and optimise the gateway diversity scheme for a scenario with two GEO satellites consisting of 302 user beams and 50 gateways. The requirements for the design of a diversity network were discussed and several challenges related with the design of the overall ground segment were analysed. The results have shown that by combining minimisation of the backbone terrestrial links together with a satellite design that minimises the payload complexity, we can reduce the ratio of redundant to active gateways as well as the backbone terrestrial links cost and hence provide a more total cost effective solution.

\section{Acknowledgment}

The authors would like to thank the partners in the EU FP7 project BATS (Broadband Access via integrated Terrestrial \& Satellite systems) for their inputs to the more recent studies that are given herein.

\section{References}

${ }^{1}$ ESA ARTES 1 Study, “TERABIT/s - Approaching the terabit/s satellite, a system study led by Astrium,” http://telecom.esa.int/ telecom/www/object/index. cfm? fobjectid=31321.

${ }^{2}$ ESA ARTES 1 Study, “TERASAT - Approaching the terabit/s satellite, a system study led by Thales Alenia Space," http: //telecom. esa.int/telecom/www/object/index.cfm?fobjectid=31254.

${ }^{3}$ Kyrgiazos, A., Evans, B., Thompson, P., and Jeannin, N., "Gateway diversity scheme for a future broadband satellite system," Advanced Satellite Multimedia Systems Conference (ASMS) and 12th Signal Processing for Space Communications Workshop (SPSC), 2012 6th, 2012, pp. 363-370.

4"Broadband Access via integrated Terrestrial and Satellite systems; European Union 7th Framework Programme," http://www. batsproject.eu/, 2012.

${ }^{5}$ Skinnemoen, H., "Gateway Diversity in Ka-band Systems," 4th Ka band Broadband Communications, Navigation and Earth Observation Conference, Venice, Italy, November 1998.

${ }^{6}$ Jeannin, N., Castanet, L., Radzik, J., Bousquet, M., Evans, B., and Thompson, P., "Smart gateways for terabit/s satellite," International Journal of Satellite Communications and Networking, Vol. 32, No. 2, 2014, pp. 93-106.

${ }^{7}$ Fernandez, M. and Williams, S., "Closed-Form Expression for the Poisson-Binomial Probability Density Function," Aerospace and Electronic Systems, IEEE Transactions on, Vol. 46, No. 2, April 2010, pp. 803-817.

${ }^{8}$ Prez-Trufero, J., Evans, B., Dervin, M., Garnier, B., and Baudoin, C., "High Throughput Satellite System with Q/V-band gateways and its integration with terrestrial broadband communication networks," 32th AIAA International Communications Satellite Systems Conference (ICSSC2014), International Communications Satellite Systems Conferences (ICSSC), American Institute of Aeronautics and Astronautics, August 2014.

${ }^{9}$ Russo, E., "Implementation of a space diversity system for Ka-band satellite communications," Communications, 1993. ICC '93 Geneva. Technical Program, Conference Record, IEEE International Conference on, Vol. 3, 1993, pp. 1468-1474 vol.3.

${ }^{10}$ ITU-R P.1623-1, "Prediction method of fade dynamics on Earth-space paths," 2005. 